\title{
Perdas Auditivas Induzidas pelo Ruído no Ambiente Ocupacional da Construção Civil
}

\author{
Bruno de Sousa Teti ${ }^{1}$ (D) orcid.org/0000-0003-4881-5287 \\ Felipe Mendes da Cruz ${ }^{1}$ (D) orcid.org/0000-0002-0163-465X \\ Eliane Maria Gorga Lago 1 (id orcid.org/0000-0003-0987-3492 \\ Tomi Zlatar ${ }^{1}$ orcid.org/0000-0002-8915-908X \\ Béda Barkokébas Júnior ${ }^{1}$ orcid.org/0000-0002-3130-3277
}

${ }^{1}$ Escola Politécnica de Pernambuco, Universidade de Pernambuco, Recife, Brasil,

E-mail do autor principal: Bruno Teti,bruno teti@hotmail.com

\section{Resumo}

O ruído é um tipo de som que provoca uma sensação auditiva desagradável. Ele pode interferir na percepção de outros sons, principalmente nos da fala. O objetivo deste trabalho foi verificar as Perdas Auditivas Induzidas pelo Ruído (PAIR) em trabalhadores da construção civil submetidos a níveis de Ruído acima do permitido pela norma brasileira NR-15 e quais as variáveis que influenciam diretamente essa problemática. A pesquisa consistiu numa revisão bibliográfica. Os artigos encontrados foram pesquisados no Portal de Periódicos CAPES/MEC, com acesso às bases de dados PubMed/MEDLINE, Science Direct, Scopus, Web of Science. As palavras chave utilizadas na busca foram: "hearing loss", "construction", "construction site", "noise" e "personal protective equipment". Inicialmente, foram encontrados 163 artigos, porém aplicando os critérios de exclusão levaram à 24 potenciais estudos. Após a leitura completa dos mesmos, 10 artigos foram incluídos para revisão detalhada. Em $90 \%$ dos estudos analisados foram apresentados resultados sugestivos de PAIR. Desta forma, foi possível concluir que as perdas auditivas são um problema nos canteiros de obras e estão diretamente ligadas as variáveis: intensidade do ruído, tempo de exposição, idade, medidas de proteção coletiva e medidas de proteção individual.

Palavras-Chave: Construção; Canteiro De Obra; PAIR; Equipamento De Proteção Individual.

\section{Abstract}

Noise is a type of sound that causes an unpleasant hearing sensation. It can interfere with the perception of other sounds, especially those of speech. The objective of this study was to verify the Noise-induced Hearing Loss (PAIR) in construction workers submitted to noise levels above the Brazilian standard NR-15 and which variables directly influence this problem. The research consisted of a bibliographical review. The articles found were searched in the Portal of Periodicals CAPES / MEC, with access to PubMed / MEDLINE databases, Science Direct, Scopus, Web of Science. The keywords used in the search were: "hearing loss", "construction", "construction site", "noise" and "personal protective equipment". Initially, 163 articles were found, but applying the exclusion criteria led to 24 potential studies. After the complete reading, 10 articles were included for detailed review. In 90\% of the studies analyzed, results suggestive of NIHL were presented. In this way, it was possible to conclude that hearing loss is a problem in construction sites and the variables are directly related: noise intensity, exposure time, age, collective protection measures and individual protection measures.

Key-words: Construction; Construction Site; NIHL; Personal Protective Equipment. 


\section{Introdução}

O ruído é um tipo de som que provoca sensação auditiva desagradável podendo interferir na percepção de outros sons, principalmente os da fala. Os efeitos nocivos no ser humano dependem da intensidade e duração da exposição, sendo que a exposição ao mesmo em excesso pode lesar extensão considerável das vias auditivas, desde a membrana timpânica até regiões do sistema nervoso central[1]. Para quantificar esse agente e proteger o trabalhador, é necessário compreender qual tipo de ruído está sendo analisado. Por tanto, segundo a NR15[3], existem dois tipos de medição do ruído, o contínuo, medido por decibéis na curva de ponderação $A \mathrm{~dB}(A)$, e o de impacto, decibéis na curva de ponderação $C \mathrm{~dB}(C)$, entende-se por ruído de impacto aquele que apresenta picos de energia acústica de duração inferior a 1 (um) segundo, a intervalos superiores a 1 (um) segundo; e ruído contínuo é tudo que não estiver compreendido na definição de impacto.

Cada medição é feita com instrumento de nível de pressão sonora, para ruído contínuo, no circuito de compensação " $A$ " e circuito de resposta lenta (SLOW); e, para ruído de impacto, com medidor de nível de pressão sonora operando no circuito linear e circuito de resposta rápida, entretanto se não houver esse equipamento é utilizado o mesmo instrumento para ruído contínuo com leitura rápida (FAST) e circuito de compensação " $\mathrm{C}$ "[2].

A NR-15[2], em seu anexo no 1, define o limite de tolerância relacionado com a natureza e o tempo de exposição ao agente, que não causará danos à saúde do indivíduo exposto durante sua vida laboral. Para uma jornada de trabalho diária de 8 (oito) horas, o nível de exposição ao ruído contínuo permitido está fixado em $85 \mathrm{~dB}(\mathrm{~A})$. Na construção civil, os níveis desse agente, por muitas vezes, encontram-se elevados devido as suas atividades e aos equipamentos utilizados, logo é necessário a utilização de Equipamento de Proteção Individual (EPI), visto que esse dispositivo tem por finalidade diminuir os riscos existentes no meio e proteger contra o surgimento de possíveis doenças causadas pelas condições de trabalho[3].

Toda via, existem algumas variáveis que intensificam a PAIR, quando o ambiente laboral não é devidamente projetado. Para Mur[4], existe uma associação entre a idade dos trabalhadores e o tempo de trabalho quanto mais avançado forem os dois maior será a existência de PAIR. Porém, não é possível, nesse estudo, relacionar a perda auditiva com os gêneros, pois os artigos têm como vieses o pequeno número de amostra de pessoas do sexo feminino[1,4-13].

O objetivo do presente trabalho foi conduzir uma revisão sistemática, descritas nos artigos na área de engenharia entre os anos de 2005 a 2017, para verificar as perdas auditivas em trabalhadores da construção civil submetidos a níveis de ruído acima do permitido pela norma brasileira NR-15.

\section{Métodos}

A metodologia, exploratória bibliográfica, utilizada para a realização dessa pesquisa seguiu as orientações dos itens de Relatório Preferidos para Revisões Sistemáticas e Meta-análises (PRISMA) [11]. Os artigos encontrados foram pesquisados no Portal de Periódicos CAPES/MEC, com acesso a bases de dados como PubMed/MEDLINE, Science Direct, Scopus, Web of Science. As palavras-chave definidas em inglês, por motivo de abrangência, foram: "Hearing loss", "Construction", "Noise", "Construction site" e "Personal Protective Equipment", utilizando a combinação dessas palavras-chave com o descritor booleano "OR", selecionando as pesquisas "No título" e "No assunto" oferecidas pela ferramenta CAPES/MEC. Adicionalmente foi feita a revisão das referências dos artigos selecionados e incluídos nessa revisão.

\subsection{Critérios de exclusão}

Os artigos encontrados foram excluídos pelo filtro de "idioma" no site da capes para seleção dos artigos (limitando aos idiomas português, inglês e espanhol). Os artigos excluídos previamente foram os que analisavam apenas as fontes de ruídos e desprezavam as consequências nos trabalhadores ou que abordavam o ruído não como um agente nocivo à saúde, mas suas formas de criação e propagação. O terceiro critério foi a data de publicação, restringindo de 2005 a 2017.

\subsection{Critérios de inclusão}

Foram incluídos os artigos que tiveram procedimentos de aferição dos níveis de ruído em 
laboratório ou em campo com objetivos de destacar a importância do controle desse agente na atividade laboral para a conservação do aparelho auditivo e as potenciais consequências no organismo do trabalhador.

\subsection{Análise de dados}

Os critérios analisados nos artigos selecionados foram: idade, sexo, utilização de Equipamento de Proteção Individual (EPI), níveis das avaliações da frequência do ruído, procedimentos prévios, períodos de teste, viés da pesquisa, critérios de exclusão, existência de programa de conservação auditiva, realização de exames audiométricos e resultado dos ouvidos avaliados.

\subsection{Resultados}

Foram encontrados 163 artigos, distribuídos na plataforma PubMed, Scopus e nas referências dos artigos selecionados após os critérios de inclusão. Aplicando o filtro "idioma", a pesquisa reduziu o quantitativo para 117 artigos possíveis de passar por a próxima fase da análise. Considerando os critérios de exclusão e inclusão, restaram apenas 24 artigos completos avaliados para elegibilidade. Ao final da leitura completa dos 24 artigos, foram selecionados 10 artigos incluídos nessa revisão, ilustrado pelo fluxograma na figura $1[1,5,7-13]$.
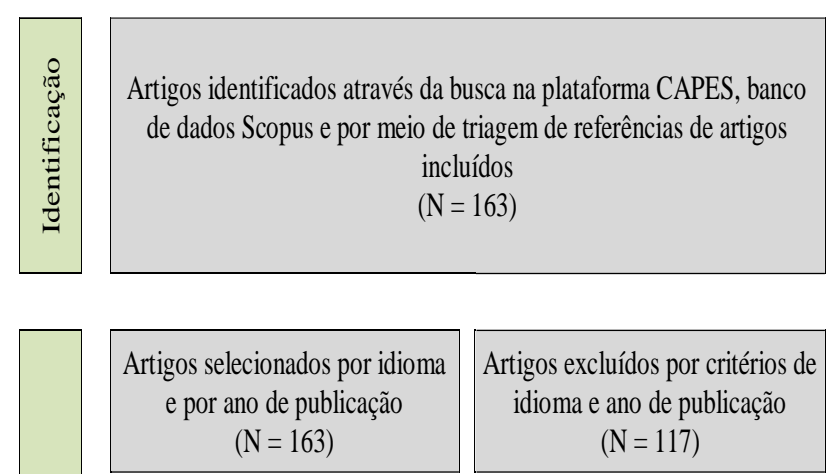

Artigos excluídos por critérios de idioma e ano de publicação $(\mathrm{N}=117)$

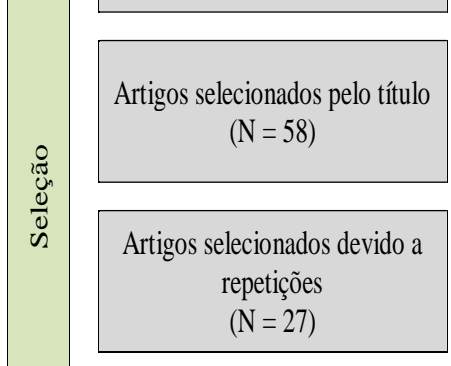

Artigos excluídos pelo título $(\mathrm{N}=31)$
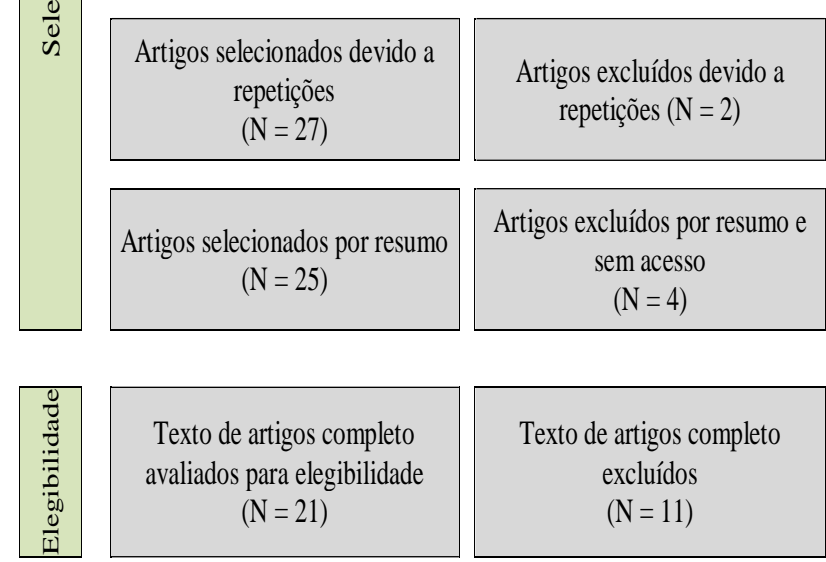

Texto de artigos completo avaliados para elegibilidade $(\mathrm{N}=21)$
Texto de artigos completo excluídos $(\mathrm{N}=11)$

Número de artigos incluídos na síntese qualitativa desta revisão $(\mathrm{N}=10)$

Figura 1 - Fluxograma da pesquisa sistematizada. Fonte: Os autores.

O quadro 1 descreve as características dos voluntários, do estudo selecionado, dos níveis da frequência de ruídos avaliados e dos vieses de cada experimento. A idade dos participantes variou entre 18 e 74 anos. Nos artigos analisados, o período de teste teve uma variação de 1 dia até 10 anos. O nível de frequência do ruído apresentou variação de $0,25 \mathrm{kHz}$ a $8,0 \mathrm{kHz}$.

$\mathrm{O}$ quadro 2 apresenta os resultados referentes às Perdas Auditivas Induzidas pelo Ruído (PAIR) e sua correlação com a ausência de medidas preventivas, fornecimento de Equipamento de Proteção Individual (EPI) e capacitação dos trabalhadores para uso dos equipamentos. 
Quadro 1 - Principais características dos estudos selecionados.

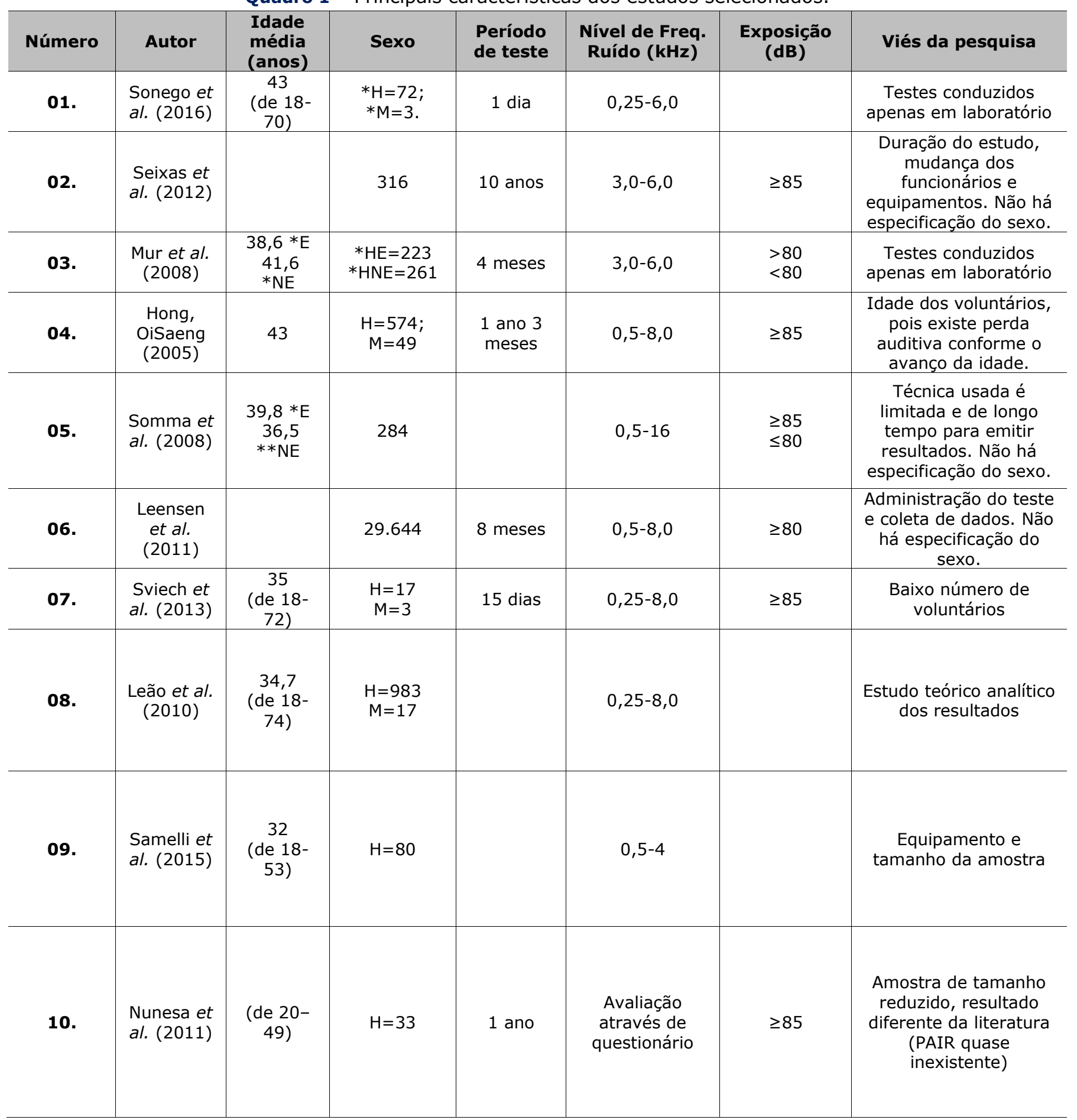

* Significado das abrevações: H (homem), M (mulher), E (expostos), NE (não expostos) 
Quadro 2 - Efeitos da exposição a níveis elevados de ruído.

\begin{tabular}{|c|c|c|c|c|}
\hline Número & Exposição & $\begin{array}{l}\text { Realização de } \\
\text { exames } \\
\text { audiométricos } \\
(\%)\end{array}$ & $\begin{array}{l}\text { Resultados } \\
\text { sugestivos de } \\
\text { Perda Auditiva } \\
\text { Induzida por } \\
\text { Ruído (PAIR) } \\
\end{array}$ & Considerações do autor \\
\hline 01. & & $76 \%$ & $46,1 \%$ & $\begin{array}{l}\text { Maior efetividade e atenuacao do protetor auricular } \\
\text { após orientacao adequada do fonoaudiologo. }\end{array}$ \\
\hline 02. & $\geq 85 \mathrm{~dB}$ & $100 \%$ & $\begin{array}{l}2 \mathrm{~dB}-3 \mathrm{~dB} \text { para os } \\
\text { funcionários em } 10 \\
\text { anos }\end{array}$ & $\begin{array}{c}\text { O estudo evidencia danos à audição quando o sujeito } \\
\text { se encontra exposto a ruído próximo de } 85 \mathrm{~dB}(\mathrm{~A}) \text { em } \\
\text { uma jornada de trabalho não inferior a } 8 \text { horas e } \\
\text { sem EPI }\end{array}$ \\
\hline 03. & $\begin{array}{l}>80 \mathrm{~dB} \\
<80 \mathrm{~dB}\end{array}$ & $100 \%$ & $\begin{array}{l}20,7 \% \\
8,4 \%\end{array}$ & $\begin{array}{l}\text { A adoção de medidas preventivas efetivas no local } \\
\text { de trabalho pode impedir a progressão da PAIR }\end{array}$ \\
\hline 04. & $\geq 85 \mathrm{~dB}$ & $100 \%$ & $>60 \%$ & $\begin{array}{l}\text { Trabalhadores com um uso mais regular do EPI } \\
\text { tiveram um resultado significativamente melhor }\end{array}$ \\
\hline 05. & $\begin{array}{l}\geq 85 \mathrm{~dB}(\mathrm{~A}) \\
\leq 80 \mathrm{~dB}(\mathrm{~A})\end{array}$ & $100 \%$ & $\begin{array}{l}\text { Elevada perda dos } \\
\text { funcionários nas } \\
\text { frequências } 14-16 \\
\mathrm{kHz}\end{array}$ & $\begin{array}{c}\text { Audiometria de alta frequencia é mais sensível e } \\
\text { deve ser utilizada como medida preventiva }\end{array}$ \\
\hline 06. & $\geq 80 \mathrm{~dB}$ & $100 \%$ & $\begin{array}{l}\text { Elevada perda dos } \\
\text { funcionários nas } \\
\text { frequências } 3-6 \mathrm{kHz}\end{array}$ & $\begin{array}{l}\text { os trabalhadores da construção holandeses exibem } \\
\text { maiores perdas auditivas do que o esperado }\end{array}$ \\
\hline 07. & $\geq 85 \mathrm{~dB}$ & & $10 \%$ & $\begin{array}{l}\text { Os protetores auriculares estudados tiveram seus } \\
\text { escores cotados em níveis aceitáveis, sendo } \\
\text { considerados ambos confortáveis. }\end{array}$ \\
\hline 08. & & $100 \%$ & $\begin{array}{c}13,2 \% \text { orelha direita } \\
\text { (31,8\% sugestiva de } \\
\text { PAIR); } \\
15,5 \% \text { orelha } \\
\text { esquerda( } 36,77 \% \\
\text { sugestiva de PAIR) }\end{array}$ & $\begin{array}{l}\text { Sugestiva de Perda Auditiva Induzida por Níveis de } \\
\text { Pressão Sonora elevados(PAINPSE), com predomínio } \\
\text { maior de perdas auditivas na orelha esquerda. } \\
\text { Ausência de uso inadequado }\end{array}$ \\
\hline 09. & & $100 \%$ & & $\begin{array}{l}\text { o grupo que recebeu treinamento para colocação } \\
\text { adequada do protetor auditivo apresentou valores de } \\
\text { atenuação maiores do que o grupo sem treinamento. }\end{array}$ \\
\hline 10. & $\geq 85 \mathrm{~dB}$ & $100 \%$ & $0,16 \%$ & $\begin{array}{l}\text { Necessidade de um Programa de controle auditivo } \\
\text { (PCA) em empresas cujos trabalhadores estão } \\
\text { expostos ao ruído, a fim de minimizar ou mesmo } \\
\text { anular os seus efeitos nocivos à saúde geral do } \\
\text { trabalhador. }\end{array}$ \\
\hline
\end{tabular}




\subsection{Discussão}

O objetivo desse estudo foi verificar as Perdas Auditivas em trabalhadores da construção civil submetidos a níveis de Ruído acima do permitido pela norma brasileira NR-15. A análise dos artigos permitiu constatar que o ruído originado devido às atividades e uso de máquinas trazem resultados sugestivos de PAIR sendo possível relacionar o grau de incidência a partir do gênero, idade e do tempo de exposição[1,4,5,7-9,12-13]. Os autores consultados apontam que apesar dos equipamentos de proteção serem fornecidos, em sua grande maioria, de acordo com a necessidade de cada atividade no campo laboral, a ausência de capacitação e fiscalização dos empregados gera não conformidades dentro das obras com consequências para saúde do trabalhador.

Seixas et al.[7] investigaram a influência do ruído acima de $85 \mathrm{~dB}(\mathrm{~A})$ na saúde do sujeito exposto em uma jornada de trabalho não inferior a 8 horas e sem utilização do equipamento de proteção individual. Para comprovar o que é informado por norma[2], foram feitos exames audiométricos em todos os trabalhadores e obteve-se um resultado final de perda auditiva nos mesmos, submetidos a essa situação. Em um período de 10 anos, todos foram afetados em sua audição com uma perda entre $2 \mathrm{~dB}$ a $3 \mathrm{~dB}$. Vale salientar que essa pesquisa tem alguns vieses. Devido ao longo período de investigação ocorreram mudanças de funcionários e dos equipamentos usados no processo de trabalho. Logo, é possível inferir que as perdas médias encontradas poderiam ser superiores, se conservados os pontos iniciais.

Entretanto, não basta apenas fornecer o EPI, segundo a análise de Sonego et al.[3], em um experimento feito com um protetor auricular tipo espuma moldável, ficou evidenciada a necessidade de orientação adequada de um profissional capacitado, nesse caso um fonoaudiólogo. Pois após análise dos riscos e a definição do devido equipamento a ser utilizado, foi desenvolvido o estudo para investigar 0 conhecimento dos empregados em relação ao manuseio do protetor auricular tipo espuma moldável. Duas averiguações de níveis de ruídos com a utilização do EPI foram feitas nesse experimento; a primeira foi entregando o equipamento e permitindo o uso do mesmo sem as devidas recomendações e a segunda foi medir os níveis de ruído após recomendações profissionais. 0 resultado dessa pesquisa revelou que o segundo experimento forneceu uma redução que varia entra 5 $\mathrm{dB}$ a $10 \mathrm{~dB}$, devido a orientação adequada. Foi possível concluir que com um trabalho adequado os $46,1 \%$ dos funcionários da empresa, que tiveram resultados sugestivos de PAIR, poderiam ter seus problemas minimizados caso fossem orientados desde o início.

As perdas auditivas induzidas por ruído são frequentes na Indústria da Construção Civil e considerando a suscetibilidade do organismo humano, aumenta a dificuldade de prevenção coletiva nos trabalhadores. Desta forma, com o intuito de encontrar medidas de prevenção para os trabalhadores através dos exames, Somma et al. [6] analisaram as perdas auditivas dos funcionários de uma empresa utilizando estudos em baixa, média e alta frequência. Os resultados obtidos foram favoráveis, pois foi possível perceber que a audiometria de alta frequência, $14-16 \mathrm{kHz}$, possui maior sensibilidade para identificação de perdas auditivas. Contudo, para execução deste método existe uma limitação em dois aspectos: o primeiro é a demora no fornecimento dos resultados; e a segunda é a necessidade de mão de obra qualificada para manuseio.

Diante das dificuldades de controle dos problemas relacionados a audição do ser humano, Mur et al.[4] trabalharam com grupos na construção civil: os expostos; e os não expostos. Ao final do experimento, verificou-se que ambos os grupos obtiveram resultados sugestivos de PAIR, 20,7\% e $8,4 \%$, respectivamente. Analisando as amostras estudadas, ficou comprovado que a audição humana vai se enfraquecendo com o passar do tempo, logo a idade torna-se uma variável susceptível e desafiadora para distinguir as perdas auditivas ocupacionais das perdas auditivas naturais. Portanto, é necessário avaliar os sujeitos e as condições de trabalho constantemente para detectar as possíveis causas dessa problemática. Uma característica que pode interferir nessa investigação é o fato de ter sido feita apenas em laboratório.

É exposto, através dos artigos incluídos, a predominância do sexo masculino em relação aos problemas investigados, isso se deve à alta exigência de vigor físico das atividades praticadas na ICC. 


\section{Conclusões}

O desenvolvimento deste trabalho possibilitou a oportunidade de extensão do assunto para um melhor entendimento, dois temas com caráter preventivo serão propostos para um maior aprofundamento. O primeiro é o estudo da influência dos níveis de alta frequência na percepção das perdas auditivas induzidas por ruído, analisando os funcionários de uma determinada empresa e comparando os resultados com as análises anteriores para concluir se o método proposto é mais eficaz na identificação da PAIR. A segunda sugestão é avaliar e identificar as perdas auditivas em uma amostra representativa com pessoas de idade avançada e relacionar os resultados obtidos com as perdas que ocorrem nos trabalhadores expostos ao ruído, com a mesma faixa etária. Desta forma, será possível estimar os problemas sugestivos de PAIR, para funcionários da construção civil, afetados pela exposição.

Todos os artigos incluídos para estudo colaboram com o objetivo dessa pesquisa, pois mostram que os trabalhadores da construção civil possuem PAIR quando as empresas que os contratam não possuem um eficiente sistema de segurança do trabalho para identificar, avaliar e controlar os riscos existentes no canteiro.

Contudo, vale salientar que os níveis dessas perdas são distintos devido a algumas variáveis encontradas nesse trabalho: idade, tempo de exposição, intensidade do ruído, medidas de proteção coletiva e medidas de proteção individual.

\section{Referências}

[1]. LEÃO, R.N.; DIAS F.A.M. Perfil Audiométrico de Indivíduos Expostos ao Ruído Atendidos no Núcleo de Saúde Ocupacional de um Hospital do Município de Montes Claros. Revista CEFAC, Minas Gerais, v.12, n.2, p. 242249, 2010.

[2]. BRASIL, NORMA REGULAMENTADORA No 15, de 05 de outubro de 2017. Atividades e operações insalubre, anexo N.० 1 . Disponível em: $<$ http://trabalho.gov.br/seguranca-e-saude-no152 trabalho/normatizacao/normas-

regulamentadoras/norma-regulamentadora-n-

15-atividades-e-operacoes-insalubres $>$. Acesso em 03 novembro. 2017.

[3]. SONEGO, M.T.; SANTOS FILHA, V.A.V. dos; MORAES A.B. de; Personal Protective Equipment. Revista CEFAC, Minas Gerais, v. 18, n. 3, p. 667-676, 2016.

[4]. MUR, P.G.; BERMÚDEZ, B.P.; MONROY, A.M. HEARING LOSS RELATED WITH NOISE EXPOSURE IN CONSTRUCTION WORKERS. Revista Medicina y Seguridad del Trabajo, Madrid, v. 54, n. 213, p. 121-130, 2008.

[5]. MAIA, P.A. ESTIMATIVA DE EXPOSIÇÕES NÃO CONTÍNUAS A RUÍDO: Desenvolvimento de um método e validação na Construção Civil. Campinas: FUNDACENTRO, 2002, 223 p.

[6]. SOMMA, G. et al. Extended HighFrequency Audiometry and Noise Induced Hearing Loss in Cement Workers. American Journal of Industrial Medicine, Estados Unidos, v. 51, n. 6, p. 452-462, 2008.

[7]. SEIXAS, N.S. et al. 10-Year prospective study of noise exposure and hearing damage among construction workers. Occupational and Environmental Medicine, Londres, v. 7, n. 2, p. 643-650, 2012.

[8]. LEENSEN, M.C.J.; DUIVENBOODEN, J.C. V.; DRESCHLER, W.A. A retrospective analysis of noise-induced hearing loss in the Dutch construction industry. International Archives of Occupational and Environmental Health, Amsterdam, v.15, n. 7, p. 577-590, 2011.

[9]. LIBERATI, A. et al. The PRISMA Statement for Reporting Systematic Reviews and Meta-Analyses of Studies That Evaluate Health Care Interventions: Explanation and Elaboration. PLoS Medicine, v. 6, n. 7, 28 p. 2009.

[10]. SAMELLI, A.G. et al. Training on hearing protector insertion improves noise attenuation. CoDAS, São Paulo, v. 8, n.3, p-514-519, 2015.

[11]. NUNES, C.P. et al. Sintomas auditivos e não auditivos em trabalhadores expostos ao ruído. Revista baiana de saúde pública, Bahia, v. 35, n. 3, p. 548-555, 2011.

[12]. SVIECH, P.S.; GONÇALVES, C.G.D.O.; MORATA, T.C.; MARQUES, J.M. Avaliação do conforto do protetor auditivo individual numa intervenção para prevenção de perdas auditivas.

http://dx.doi.org/10.25286/repa.v4i1.971 
Revista CEFAC, Minas Gerais, v. 15, n.5, p. 1325-1337, 2013.

[13]. Hong O. Hearing loss among operating engineers in American construction industry. International Archives of Occupational and Environmental Health, Amsterdam, v. 9, n. 3, p. 565-574, 2005.

[14]. Coordenação de Aperfeiçoamento Pessoal de Nível Superior. Portal Periódicos CAPES. Coordenação de Aperfeiçoamento Pessoal de Nível Superior.

http://www.periodicos.capes.gov.br. Published 2017. AccessedJanuary 6, 2018. 